\title{
PROPERTIES OF SELF SIMILAR SOLUTIONS OF REACTION-DIFFUSION SYSTEMS OF QUASILINEAR EQUATIONS
}

\author{
D. K. MUHAMEDIYEVA \\ Research Scholar Tashkent University of Information Technologies Named after \\ Muhammad al-Khwarizmi Scientific and Innovation Center of Information and
}

Communication Technologies, Tashkent, Uzbekistan

\begin{abstract}
In this paper, considered a parabolic system of two quasi linear reaction-diffusion equations with the source and absorption task and the properties of self-similar solutions of a system of quasi linear reaction-diffusion equations for the source and absorption task. Self-similar system of equations is constructed by the method of nonlinear splitting. Estimates of the solutions and the free boundary that arises in this case are found, which makes it possible to choose suitable initial approximations for each value of the numerical parameters.

KEYWORDS: Source and Absorption Equation, Parabolic System, Quasi Linear Equations, Self-Similar System of Equations \& Reaction-Diffusion
\end{abstract}

Received: Jan 02, 2018; Accepted: Jan 23, 2018; Published: Mar 08, 2018; Paper Id.: IJMPERDAPR201864

\section{INTRODUCTION}

Study of nonlinear mathematical models of various physical, biological, chemical and other phenomena and processes is one of the important directions of mathematical modeling. As examples, we note such physical theories as non-linear quantum mechanics, nonlinear electrodynamics and optics, non-linear theory of plasmas, nonlinear acoustics, nonlinear conduction, nonlinear diffusion, and other theories based on mathematical models which are nonlinear differential equations in partial derivatives. The study of the linear mathematical models of physical processes are easy to study, since the underlying linear differential equations developed general methods for their solution [1,2]. In applied tasks, the actual physical processes are nonlinear and for their adequate description, one should use nonlinear mathematical models.

Nonlinear models of mathematical physics, describing phenomena and processes in a wider range of physical parameter changes and have a better capacity of information about these phenomena and processes. Linear models are typically special cases of nonlinear models. They can give only an approximate picture of the phenomenon under study without identifying the observed effects. Studies show that the nonlinearities change not only the quantitative characteristics of the processes, but the qualitative picture of their behavior. Interestingly, from the point of view of applications to study the following classes of nonlinear differential equations in which the unknown function and the derivative of this function consists of exponential way. Then, with the comparison theorems of solutions of this class can be extended. These types of nonlinearities are often encountered in problems of the theory of filtration, diffusion, thermal conductivity, magnetohydrodynamics, biological populations, the oil industry, etc. $[3,4,5,6]$. 
These models more accurately describe the physics of the process and, therefore, their research shows that there are new effects related to the nonlinearity of the studied process. So was found the effects of the finite speed of propagation of perturbations [1], localization of solutions and different modes of processes. The first effect of finite speed of propagation of perturbations was obtained and applied to the problem of nonlinear thermal conductivity, in the work of Zeldovich and A. S. Kompaneetz[1], for the problem of nonlinear filtering in the work of G. I. Barenblatt[2,3], which are independently from each other, and got this effect. Further mathematical questions were studied in works of O. A. Oleinik, A. S. Kalashnikov [5,6,7], A. A. Samarskiy and his students [2,3-4], B. Knerr [3].

\section{Statement of the Problem}

In the field $\mathrm{Q}=\{(\mathrm{t}, \mathrm{x}): 0<\mathrm{t}<\infty, \mathrm{x} \in \mathrm{R}\}$ considers a parabolic system of two quasilinear equations of source and absorption

$$
\begin{aligned}
& \left\{\begin{array}{l}
\frac{\partial u_{1}}{\partial t}=\frac{\partial}{\partial x}\left(D_{1} u_{1}^{\sigma_{1}} \frac{\partial u_{1}}{\partial x}\right)+k_{1}(t) u_{1} \cdot\left(1-u_{2}^{\beta_{1}}\right) \\
\frac{\partial u_{2}}{\partial t}=\frac{\partial}{\partial x}\left(D_{2} u_{2}^{\sigma_{2}} \frac{\partial u_{2}}{\partial x}\right)+k_{2}(t) u_{2} \cdot\left(1-u_{1}^{\beta_{2}}\right)
\end{array}\right. \\
& \left.u_{1}\right|_{t=0}=u_{10}(x),\left.u_{2}\right|_{t=0}=u_{20}(x)
\end{aligned}
$$

Which describes the process of heat distribution in a nonlinear two-component environment, the diffusion coefficient of which is equal to $D_{1} u_{1}^{\sigma_{1}}$ and $D_{2} u_{2}^{\sigma_{2}}, \sigma_{1}, \sigma_{2}, \beta_{1}, \beta_{2}$ - positive real numbers, $u_{1}=u_{1}(t, x) \geq 0$, $u_{2}=u_{2}(t, x) \geq 0$ - desired solution.

Estimates of solutions are found, which makes it possible to choose suitable initial approximations for each value of numerical parameters.

\section{Construction of a Self-Similar System of Equations}

Self-similar system of equations is constructed by the method of nonlinear splitting[4].

$$
\begin{aligned}
& \left\{\begin{array}{l}
\frac{d}{d \xi}\left(f_{1}^{\sigma_{1}} \frac{d f_{1}}{d \xi}\right)+\frac{\xi}{2} \frac{d f_{1}}{d \xi}+\mu_{1}\left(f_{1}-f_{1} f_{2}^{\beta_{1}}\right)=0, \\
\frac{d}{d \xi}\left(f_{2}^{\sigma_{2}} \frac{d f_{2}}{d \xi}\right)+\frac{\xi}{2} \frac{d f_{2}}{d \xi}+\mu_{2}\left(f_{2}-y_{2} f_{1}^{\beta_{2}}\right)=0,
\end{array}\right. \\
& \text { where } \quad \mu_{i}=\frac{1}{\left(1-\gamma_{i} \sigma_{i}\right)},
\end{aligned}
$$

To construct the upper solution of the system (1) - (2), we introduce functions

$$
\begin{aligned}
& \bar{f}_{1}(\xi)=A\left(a-\xi^{2}\right)_{+}^{{ }^{n_{1}}}, \bar{f}_{2}(\xi)=B\left(a-\xi^{2}\right)_{+}^{{ }^{n_{2}}}, \\
& \text { where } n_{1}=-\frac{1}{\sigma_{1}}, n_{2}=-\frac{1}{\sigma_{2}}, \quad(b)_{+}=\max (0, b),
\end{aligned}
$$




$$
\begin{aligned}
& u_{1}(t, x) \leq u_{1+}(t, x)=e^{-\int_{0}^{t} k_{1}(\eta) d \eta} \overline{f_{1}}(\xi), \\
& u_{2}(t, x) \leq u_{2+}(t, x)=e^{-\int_{0}^{t} k_{2}(\eta) d \eta} \bar{f}_{2}(\xi), \\
& \bar{f}_{1}(\xi), \bar{f}_{2}(\xi) u \quad \tau(t) \text {-the functions defined above. }
\end{aligned}
$$

\section{Cross-Diffusion System of Two Quasilinear Reaction-Diffusion Equations of the Source and Absorption}

In the domain $\mathrm{Q}=\{(\mathrm{t}, \mathrm{x}): 0<\mathrm{t}<\infty, \mathrm{x} \in \mathrm{R}\}$ considered a parabolic system of two quasilinear reaction-diffusion equations for the source and absorption

$$
\begin{gathered}
\left\{\begin{array}{l}
\frac{\partial u_{1}}{\partial t}=\frac{\partial}{\partial x}\left(D_{1} u_{2}^{\sigma_{1}} \frac{\partial u_{2}}{\partial x}\right)+k_{1}(t) u_{1}\left(1-u_{2}^{\beta_{1}}\right), \\
\frac{\partial u_{2}}{\partial t}=\frac{\partial}{\partial x}\left(D_{2} u_{1}^{\sigma_{2}} \frac{\partial u_{1}}{\partial x}\right)+k_{2}(t) u_{2}\left(1-u_{1}^{\beta_{2}}\right),
\end{array}\right. \\
\left.u_{1}\right|_{t=0}=u_{10}(x),\left.u_{2}\right|_{t=0}=u_{20}(x),
\end{gathered}
$$

which describes the process of process of absorption in a nonlinear two-component environment, diffusion coefficient whose are $D_{1} u_{2}^{\sigma_{1}}$ and $D_{2} u_{1}^{\sigma_{2}}, \sigma_{1}, \sigma_{2}, \beta_{1}, \beta_{2}$ - positive real numbers, $u_{1}=u_{1}(t, x) \geq 0$, $u_{2}=u_{2}(t, x) \geq 0$ - sought solutions.

Let $\gamma_{2} \sigma_{1}>1, \gamma_{1} \sigma_{2}=\gamma_{2} \sigma_{1}, \sigma_{2}\left(b_{2}+1\right)+\beta_{2}\left(b_{1}+1\right)=\sigma_{1}\left(b_{1}+1\right)+\beta_{1}\left(b_{2}+1\right), c_{i}>0$. In this case, assuming in (3)

$$
w_{i}(\tau(t), x)=f_{i}(\xi), \quad \xi=|x| / \tau_{1}^{1 / 2}, i=1,2,
$$

and taking into account that the equation for $w_{i}(\tau, x)$ without lower terms always have a self-similar solution in the case $1-\gamma_{3-i} \sigma_{i} \neq 0$, obtain the system

$$
\left\{\begin{array}{l}
\frac{d}{d \xi}\left(f_{2}^{\sigma_{1}} \frac{d f_{2}}{d \xi}\right)+\frac{\xi}{2} \frac{d f_{2}}{d \xi}+\mu_{1}\left(f_{1}-f_{1} f_{2}^{\beta_{1}}\right)=0 \\
\frac{d}{d \xi}\left(f_{1}^{\sigma_{2}} \frac{d f_{1}}{d \xi}\right)+\frac{\xi}{2} \frac{d f_{1}}{d \xi}+\mu_{2}\left(f_{2}-f_{2} f_{1}^{\beta_{2}}\right)=0
\end{array}\right.
$$

where

$$
\mu_{i}=\frac{1}{1-\gamma_{3-i} \sigma_{i}}
$$

To construct the upper solution of the system (4), we introduce functions 
$\bar{f}_{1}(\xi)=A\left(a-\xi^{2}\right)_{+}^{{ }^{n_{1}}}, \bar{f}_{2}(\xi)=B\left(a-\xi^{2}\right)_{+}^{{ }^{n_{2}}}$

where

$$
\begin{aligned}
& n_{1}=\frac{\left(\sigma_{1}+2\right)}{1-\left(\sigma_{1}+1\right)\left(\sigma_{2}+1\right)}, n_{2}=\frac{\left(\sigma_{2}+2\right)}{1-\left(\sigma_{1}+1\right)\left(\sigma_{2}+1\right)},(b)_{+}=\max (0, b), \\
& \bar{f}_{2}^{\sigma_{1}} \frac{d \overline{f_{2}}}{d \xi}=-2 B^{\sigma_{1}+1} \gamma_{2} \xi \bar{f}_{1} \in C(0, \infty), \\
& \bar{f}_{1}^{\sigma_{2}} \frac{d \overline{f_{1}}}{d \xi}=-2 A^{\sigma_{2}+1} \gamma_{1} \xi \bar{f}_{2} \in C(0, \infty)
\end{aligned}
$$

and

$$
\left\{\begin{array}{l}
\frac{d}{d \xi}\left(\bar{f}_{2}^{\sigma_{1}} \frac{d \bar{f}_{2}}{d \xi}\right)=-2 \gamma_{2} B^{\sigma_{1}+1}\left(\bar{f}_{1}+\xi \frac{d \overline{f_{1}}}{d \xi}\right) \\
\frac{d}{d \xi}\left(\bar{f}_{1}^{\sigma_{2}} \frac{d \overline{f_{1}}}{d \xi}\right)=-2 \gamma_{1} A^{\sigma_{2}+1}\left(\bar{f}_{2}+\xi \frac{d \bar{f}_{2}}{d \xi}\right)
\end{array}\right.
$$

$\mathrm{A}$ and $\mathrm{B}$ are chosen from the system of nonlinear algebraic equations

$$
\begin{aligned}
& \gamma_{2} B^{\sigma_{1}+1}=1 / 2, \\
& \gamma_{1} A^{\sigma_{2}+1}=1 / 2
\end{aligned}
$$

and

$$
\beta_{1}=1 / n_{2}, \beta_{2}=1 / n_{1}
$$

Then in the domain $Q$ according to the principle of comparing solutions we have

$$
\begin{aligned}
& u_{1}(t, x) \leq u_{1+}(t, x)=e^{-\int_{0}^{t} k_{1}(\eta) d \eta} \bar{f}_{1}(\xi) \\
& u_{2}(t, x) \leq u_{2+}(t, x)=e^{-\int_{0}^{t} k_{2}(\eta) d \eta} \bar{f}_{2}(\xi) \\
& \text { where } \bar{f}_{1}(\xi), \bar{f}_{2}(\xi) \text { and } \tau(t) \text {-previously defined function. }
\end{aligned}
$$

Slow diffusion.Case $n_{1}>0, n_{2}>0, n>0$ (slow diffusion). Using the method of nonlinear splitting for the solution of the equation (4) obtained the following functions

$$
\bar{\theta}_{1}(\xi)=\left(a-\xi^{2}\right)_{+}^{{ }^{n_{1}}}, \bar{\theta}_{2}(\xi)=\left(a-\xi^{2}\right)_{+}^{{ }^{n_{2}}},
$$

where 
$n_{1}=\frac{\left(\sigma_{1}+2\right)}{n}, n_{2}=\frac{\left(\sigma_{2}+2\right)}{n}, n=1-\left(\sigma_{1}+1\right)\left(\sigma_{2}+1\right)$,

$a>0,(y)_{+}=\max (y, 0), \xi<a$. It is known that for the global existence of a solution of the task (4) of the functions $f_{i}(\xi)$ must satisfy the following inequality:

$$
\left\{\begin{array}{l}
\frac{d}{d \xi}\left(f_{2}^{\sigma_{1}} \frac{d f_{2}}{d \xi}\right)+\frac{\xi}{2} \frac{d f_{2}}{d \xi}+\mu_{1}\left(f_{1}-f_{1} f_{2}^{\beta_{1}}\right) \leq 0, \\
\frac{d}{d \xi}\left(f_{1}^{\sigma_{2}} \frac{d f_{1}}{d \xi}\right)+\frac{\xi}{2} \frac{d f_{1}}{d \xi}+\mu_{2}\left(f_{2}-f_{2} f_{1}^{\beta_{2}}\right) \leq 0
\end{array}\right.
$$

and

$$
\beta_{1}=1 / n_{2}, \beta_{2}=1 / n_{1}
$$

It is shown that the functions $\bar{\theta}_{1}(\xi), \bar{\theta}_{2}(\xi)$ will be asymptotic finite solutions.

Теорема 1. Finite solution of the problem (4) at $\xi \rightarrow a_{-}$has asymptotics $f_{i}(\xi) \sim \bar{\theta}_{i}(\xi), i=1,2$.

Fast diffusion.Case $n_{1}>0, n_{2}>0, n<0$ (fast diffusion). For (4) there is

$$
\chi_{1}(\xi)=\left(a+\xi^{2}\right)^{n_{1}}, \chi_{2}(\xi)=\left(a+\xi^{2}\right)^{n_{2}}
$$

where

$$
n_{1}=\frac{-\left(\sigma_{1}+2\right)}{n}, n_{2}=\frac{-\left(\sigma_{2}+2\right)}{n}, n=\left(\sigma_{1}+1\right)\left(\sigma_{2}+1\right)-1
$$

$a>0$.

Theorem 2. At $\xi \rightarrow+\infty$ the vanishing at infinity solution of the problem (4) has asymptotic $f_{i}(\xi) \sim \chi_{i}(\xi)$.

And also in the domain $\mathrm{Q}=\{(\mathrm{t}, \mathrm{x}): 0<\mathrm{t}<\infty, \mathrm{x} \in \mathrm{R}\}$ considered parabolic system of two quasilinear equations of the source and absorption task

$$
\left\{\begin{array}{l}
\frac{\partial u_{1}}{\partial t}=\frac{\partial}{\partial x}\left[\left(a_{11} u_{1}^{m}+a_{12} u_{2}^{m}\right) \frac{\partial u_{1}}{\partial x}+\left(b_{11} u_{1}^{m}+b_{12} u_{2}^{m}\right) \frac{\partial u_{2}}{\partial x}\right]+k_{1}(t) u_{1}\left(1-u_{2}^{\beta_{1}}\right) \\
\frac{\partial u_{2}}{\partial t}=\frac{\partial}{\partial x}\left[\left(a_{21} u_{1}^{m}+a_{22} u_{2}^{m}\right) \frac{\partial u_{1}}{\partial x}+\left(b_{21} u_{1}^{m}+b_{22} u_{2}^{m}\right) \frac{\partial u_{2}}{\partial x}\right]+k_{2}(t) u_{2}\left(1-u_{1}^{\beta_{2}}\right)
\end{array}\right.
$$

$a_{i j}, b_{i j}$ - positive real numbers, $\beta_{1}, \beta_{2} \geq 0, u_{1}=u_{1}(t, x) \geq 0, u_{2}=u_{2}(t, x) \geq 0$ - desired solution. At $a_{i j} \neq 0, b_{i j}=0$ or $a_{i j}=0, b_{i j} \neq 0$ mathematical model (5) is a system of the type reaction-diffusion with diffusion 
coefficients $a_{i j} u_{i}^{m} \geq 0, b_{i j} u_{i}^{m} \geq 0$. In the case where at least one of the coefficients $a_{i j} \neq 0$ and $b_{i j} \neq 0$ (the sign can be arbitrary), system (5) is cross-diffusion (mutual-diffusion for $\mathrm{i}, \mathrm{j}=1,2$ ).

Qualitative properties of the considered problem are studied by constructing self-similar system of equations for (4).

Then, the obtained self-similar

system $\left\{\begin{array}{l}\frac{d}{d \xi}\left[\left(a_{11} f_{1}^{m}+a_{12} f_{2}^{m}\right) \frac{d f_{1}}{d \xi}+\left(b_{11} f_{1}^{m}+b_{12} f_{2}^{m}\right) \frac{d f_{2}}{d \xi}\right]+\frac{\xi}{2} \frac{d f_{1}}{d \xi}+\psi_{1} f_{1}\left(1-f_{2}^{\beta_{1}}\right)=0, \\ \frac{d}{d \xi}\left[\left(a_{21} f_{1}^{m}+a_{22} f_{2}^{m}\right) \frac{d f_{1}}{d \xi}+\left(b_{21} f_{1}^{m}+b_{22} f_{2}^{m}\right) \frac{d f_{2}}{d \xi}\right]+\frac{\xi}{2} \frac{d f_{2}}{d \xi}+\psi_{2} f_{2}\left(1-f_{1}^{\beta_{2}}\right)=0,\end{array}\right.$

(6)

and $\tau_{1}=\tau_{1}(t)$ is chosen as

$\tau_{1}(\tau)=\left\{\begin{array}{lr}\frac{(\mathrm{T}+\tau)^{-\gamma_{1} m+1}}{-\gamma_{1} m+1}, & \text { if }-\gamma_{1} m+1 \neq 0, \\ \ln (T+\tau), & \text { if }-\gamma_{1} m+1=0, \\ (T+\tau), & \text { if } m=0,\end{array}\right.$

if $\gamma_{2} m_{1}=\gamma_{1} m_{2}$.

System (6) has an approximate solution of the form

$$
\bar{f}_{1}=A\left(a-b \xi^{2}\right)_{+}^{\eta_{1}}, \bar{f}_{2}=B\left(a-b \xi^{2}\right)_{+}^{\eta_{2}}(y)_{+}=\max (0, y) .
$$

\section{Case I}

$\eta_{1}=\eta_{2}, \eta_{1}=\frac{1}{m}$

and the coefficients A and B are determined from the solution of the systems of nonlinear algebraic equations

$$
\left\{\begin{array}{l}
{\left[a_{11} A^{m}+a_{12} B^{m}\right] \cdot \eta_{1} A+\left[b_{11} A^{m}+b_{12} B^{m}\right] \cdot \eta_{2} B \cdot(-2 b)=A,} \\
{\left[a_{21} A^{m}+a_{22} B^{m}\right] \cdot \eta_{1} A+\left[b_{21} A^{m}+b_{22} B^{m}\right] \cdot \eta_{2} B \cdot(-2 b)=B .}
\end{array}\right.
$$

\section{Case II}

$$
\begin{aligned}
& a_{11}=0 ; a_{12} \neq 0 ; b_{11}=0 ; b_{12}=0 ; \\
& a_{21}=0 ; a_{22}=0 ; b_{21} \neq 0 ; b_{22}=0 ; \\
& \eta_{2}=\frac{1}{m}, \quad \eta_{1}=\frac{1}{m} .
\end{aligned}
$$

And the coefficients A and B are determined from the solution of the system of nonlinear algebraic equations 


$$
\begin{aligned}
& a_{12} B^{m} \cdot \eta_{1}(-2 b)=1, \\
& b_{21} A^{m} \cdot \eta_{2}(-2 b)=1 .
\end{aligned}
$$

\section{Case III}

$$
\begin{aligned}
& a_{11}=0 ; a_{12}=0 ; b_{11}=0 ; b_{12} \neq 0 ; \\
& a_{21} \neq 0 ; a_{22}=0 ; b_{21}=0 ; b_{22}=0 ; \\
& \eta_{1}=\frac{m+2}{(\mathrm{~m}+1)^{2}-1}, \quad \eta_{2}=\frac{m+2}{(\mathrm{~m}+1)^{2}-1} .
\end{aligned}
$$

And the coefficients A and B are determined from the solution of the system of nonlinear algebraic equations

$$
\left\{\begin{array}{l}
b_{12} B^{m+1} \cdot \eta_{2}(-2 b)=A \\
a_{21} A^{m+1} \cdot \eta_{1}(-2 b)=B .
\end{array}\right.
$$

\section{Types of Self-Similar Regimes with Peaking}

There can be three types of self-similar modes with peaking: NS, S, and LS. At $0<\beta_{i}<m_{i}, i=1,2$ HS mode is realized. Studies have shown that a self-similar problem in this case has a unique eigenfunction that decreases monotonically on a segment with a maximum at the center of symmetry. A self-similar solution is a wave whose amplitude and front increase in the blast mode.

At $\beta_{i}=m_{i}, i=1,2$ takes place S-mode. Self-similar solution is a non-stationary dissipative localized structure. Inside the area of localization, the number of individuals increases in regime with aggravation, and beyond remains equal to zero.

Self-similar solution in LS-mode, is a non-stationary dissipative structure; all points of which move to the center of symmetry, the solution at $T=-\tau$ turns into infinity in a single point - the center of symmetry. Self-similar solutions can exist when $\beta_{i}>m_{i}, i=1,2$.

The following are the results of numerical experiments for different values of the parameters.

To determine the number of eigenfunctions (EF) self-similar problem in LS - mode, and studies of the nature of their dependence on the parameter was conducted bifurcation analysis of the solutions. The first ten eigenfunctions continued on the parameter $\beta_{i}$ for a fixed parameter $m_{i}\left(m_{i}=2\right)$. 


\begin{tabular}{|c|c|c|c|c|c|}
\hline Parameter Values & $\begin{array}{c}t=1 \\
a_{11}=1 ; a_{12}=1 ; b_{11}=1 ; b_{12}=1 \\
a_{21}=1 ; a_{22}=1 ; b_{21}=1 ; b_{22}=1\end{array}$ & $\begin{array}{c}t=40 \\
a_{11}=1 ; a_{12}=1 ; b_{11}=1 ; b_{12}=1 \\
a_{21}=1 ; a_{22}=1 ; b_{21}=1 ; b_{22}=1\end{array}$ & $\begin{array}{c}t=1 \\
a_{11}=0 ; a_{12}=1 ; b_{11}=0 ; b_{12}=0 \\
a_{21}=0 ; a_{22}=0 ; b_{21}=1 ; b_{22}=0\end{array}$ & $\begin{array}{c}t=40 \\
a_{11}=0 ; a_{12}=1 ; b_{11}=0 ; b_{12}=0 \\
a_{21}=0 ; a_{22}=0 ; b_{21}=1 ; b_{21}=0\end{array}$ & $\begin{array}{c}t=40 \\
a_{11}=0 ; a_{12}=0 ; b_{11}=0 ; b_{12}=1 \\
a_{21}=1 ; a_{22}=0 ; b_{21}=0 ; b_{22}=0\end{array}$ \\
\hline $\begin{array}{c}\text { LS-regime } \\
m_{1}=2, m_{2}=2 \\
\beta_{1}=3, \quad k_{1}=2 \\
\beta_{2}=3, \quad k_{2}=9 \\
\text { eps }=10^{-3} \\
m_{i}<\beta_{i}<\hat{\beta}_{i j} \\
\hat{\beta}_{i j}=m_{i}+m_{i} /(j-1) \\
, j=3\end{array}$ & - & 㓊 & - & & \\
\hline $\begin{array}{c}\text { S-regime } \\
m_{1}=2, m_{2}=2 \\
\beta_{1}=2, \quad k_{1}=2 \\
\beta_{2}=2, \quad k_{2}=9 \\
\text { eps }=10^{-3} \\
\beta_{i}=m_{i}\end{array}$ & - & is- & - & (- & "s- \\
\hline $\begin{array}{c}\text { S-regime } \\
m_{1}=4, m_{2}=4 \\
\beta_{1}=4, \quad k_{1}=2 \\
\beta_{2}=4, \quad k_{2}=9 \\
\text { eps }=10^{-3} \\
m_{i}=\beta_{i}\end{array}$ & | & 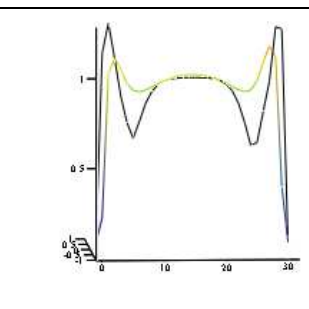 & | & & \\
\hline $\begin{array}{c}\text { HS-regime } \\
m_{1}=0.7, m_{2}=0 . \\
\beta_{1}=0.5, k_{1}=2 \\
\beta_{2}=0.5, k_{2}=3 \\
\text { eps }=10^{-3} \\
\beta_{i}<m_{i}\end{array}$ & . &. & 政 & & \\
\hline $\begin{array}{c}\text { HS-regime } \\
m_{1}=1.4, m_{2}=1.4 \\
\beta_{1}=0.5, \quad k_{1}=2 \\
\beta_{2}=0.7, \quad k_{2}=5 \\
\text { eps }=10^{-3} \\
\beta_{i}<m_{i}\end{array}$ & os- & $\because 2$ & . & .4 &. \\
\hline
\end{tabular}

Figure 1: Results of Computational Experiment

\section{CONCLUSIONS}

The analysis showed that the eigenfunction with the number $j=2,3,4, \ldots$ exists in the interval $m_{i}<\beta_{i}<\hat{\beta}_{i j}$, where 
$\hat{\beta}_{i j}=m_{i}+m_{i} /(j-1), i=1,2, j=2,3,4, \ldots$

Values $\beta_{i}=m_{i}$ и $\beta_{i}=\hat{\beta}_{i j}$ are the bifurcation points in which the EF ceases to exist. The first EF exists for any value $\beta_{i}>m_{i}, i=1,2$. It follows from (7) that when $\beta_{i}>\hat{\beta}_{i 2}=2 m_{i}, i=1,2$ self-similar task in LS mode can have only one eigen function.

The greater the number of SF, the narrower the interval of the parameter $\beta_{i}$ in which it exists.

The number of eigenfunctions $\mathrm{N}$ that has a self-similar problem for the given $\beta_{i}$ and $m_{i}$ is determined by the formula, where $a_{i}=\beta_{i} /\left(\beta_{i}-m_{i}\right), i=1,2$ :

$$
N_{i}=\left\{\begin{array}{l}
{\left[a_{i}\right], \text { if } a \text { is not an integer }} \\
a_{i}-1, \text { if } a \text { is integer }
\end{array}\right.
$$

The sequence of bifurcation points that define the right boundary of the domain of existence of eigenfunctions is an infinite sequence that converges to a point $1+=\infty \sigma \beta$, which is the common left boundary of the intervals of existence of all eigenfunctions in the LS-mode.

\section{REFERENCES}

1. Barenblatt G. I., Entov V. M., Ryzhik V. M. Theory of unsteady filtration of fluid and gas. M.: Nedra, 1972.

2. Martinson L. K. the Study of mathematical models of nonlinear heat conduction in media with volumetric absorption. - In the book: Mathematical modeling. Processes in nonlinear media. M.: Nauka, 1986, pp. 279-309.

3. Oleinik O. A., Kalashnikov A. S., Zhou Yu-Lin. The Cauchy problem and boundary problems for equations of the type of nonstationary filtration. Izv. USSR ACADEMY OF SCIENCES. Ser. Mat. 1958, vol. 22, No. 5, pp. 667-704.

4. Vazquez, Juan L., Galaktionov, V. A., Asymptotic behavior of solutions of nonlinear equations of the diffusion-absorption at a critical indicator. I/DAN SSSR, 1990, vol. 314, no. 3, pp. 530-534.

5. Galaktionov V. A. On some properties of traveling waves in a medium with nonlinear thermal conductivity and heat sources. // JVM and MF, 1981, vol. 21. No. 4, pp. 980-989.

6. Galaktionov, V. A., On conditions of non-existence and localization of solutions of the Cauchy problem for a class of nonlinear parabolic equations. // JVM and MF, 1983, V. 23. No. 6, pp. 1341-1354.

7. Alle W.C. Animal aggregation: a study in general sociology. Chicago: ChicagoUniv.Press., 1931.

8. Belotelov N. V., Locusts D. A. Linear stability analysis of systems with diffusion on environmental example. Biophysics, 1984, No. 1, pp. 130-134

9. Murray George. Nonlinear differential equations in biology. Lectures on models. Moscow, Mir, 1983, 394 p.

10. Aripov M. M. Method of standard equations for solutions of nonlinear boundary value problems. - Tashkent: FAN, 1988. $137 p$.

11. 5.Aripov M, Sadullaeva Sh. Qualitative properties of solutions of a doubly nonlinear reaction-diffusion system with a source. Journal of Applied Mathematics and Physics, 3, 2015, 1090-1099. 
12. M. M. Aripov, Z. Rakhmonov. About the asymptotics of the solutions of heat conduction problem with a source and nonlinear boundary condition. Computing technology, Volume 20 the Bulletin of KazNU. Al-Farabi, Series mathematics, mechanics and Informatics № 3 (86), Part 2, 2015, 216-223.

13. Aripov M., Matyakubov A. on the asymptotic behavior of nonlinear parabolic equations not in divergence form. Computing technology, Volume 20 the Bulletin of KazNU. Al-Farabi, Series mathematics, mechanics and Informatics № 3 (86), Part 2, $2015,275-282$.

14. Aripov M., Mukhamedieva D.K. Population Model of Kolmogorov-Fisher type with Nonlinear Cross-diffusion. Mathematics and Computers in Science and Engineering Series, 40, 2015, 316-320.

15. Aripov M.M., Muhamediyeva D.K. Population model of two competing populations with double nonlinear diffusion. International scientific and technical journal “Chemical technology. Control and management, № 3-4” and “Journal of Korea multimedia society” South Korea, Seoul, 2015, 87-92.

16. Aripov M.M., Mukhamediyeva D.K. Splitting algorithm in Kolmogorov-Fisher type reaction-diffusion task //International Journal of Mathematics and Computer Applications Research, Vol.3, № 4, 2013, p. 1-8.

17. Aripov M.M., Mukhamediyeva D.K. To the numerical modeling of self-similar solutions of reaction-diffusion system of the one task of biological population of Kolmogorov-Fisher type //International Journal of Research in Engineering and Technology, Vol.2, №11, 2013, p.281-286.

18. Aripov M.M, Muhamedieva D.K. Cross-diffusion model with double nonlinearity in hetrogenous environment //International scientific-technical journal of Chemical technology. Monitoring and control, No. 4, 2014, pp. 76-84

19. Aripov M.M, Muhamediyeva D. K. Approaches to numerical modeling of the problem of reaction with the diffusion of Kolmogorov-Fisher in the two-dimensional case //Uzbek journal problems of Informatics and energy, No. 1-2, 2014, pp. 21 26.

20. Aripov M.M, Muhamediyeva D. K. Waves in diffusive systems one task of biological population of Kolmogorov-Fisher dual nonlinear cross-diffusion //Uzbek journal problems of Informatics and energy, No. 3-4, 2015, pp. 44-51, (05.00.00; No. 5).

21. Aripov M.M, Muhamediyeva D. K. Population model convective transfer of Kolmogorov-Fisher dual nonlinear cross-diffusion I/international scientific-technical journal of Chemical technology. Control and management, № 5, 2015, p. 16-20, (05.00.00; №12).

22. Aripov M.M, Muhamediyeva D. K. Cross-diffusion model of convective transfer with double nonlinearity. //Problems computing and applied mathematics, No. 1, 2015, pp. 5-9, (05.00.00; №23)

23. Kapitsa S. P., Kurdyumov S. P., Malinetskii, G. G. Synergetics and forecasts of the future, M., 1997.

24. 17.S. P. Kurdyumov, E. S. Kurkina, "the Spectrum of eigenfunctions of the self-similar problem for the nonlinear equation of heat-conduction with a source," Zh. Vychisl. mod. and mod. Phys., $44: 9$ (2004), 1619-1637; Comput. Math. Phys., 44:9 (2004), 1539-1556.

25. Modes with peaking. The evolution of the idea. Moscow: Nauka. 1998.

26. Dmitrenko N. V., Kurdyumov S. P., Mikhailov A. P., Samarskii A. A. Localization of thermonuclear combustion in a plasma with electronic thermal conductivity //J. of Appl. 1977. T. 26. Vol.9. C. 620-624.

27. Tikhonov A. N., Samarskiy A. A. zaklyaz'minskiy L. A. etc. non-linear effect of education of self-sustaining high-temperature electrically conducting gas layer in nonstationary processes of magnetohydrodynamics // Doc. An SSSR, 1967, Vol. 173, No. 4, 808-811. 
28. V. Volterra. Mathematical theory of struggle for existence-M.: Nauka, 1976, 288 p.

29. Bazykin, A. D., Berezovskaya F. S. Allee Effect, lower critical population size and dynamics of a system a predator - victim. Problems of ecological monitoring and ecosystem modeling. - L.: Gidrometeoizdat, 1979, vol. 2, pp. 161-175.

30. Belotelov N. V., Lobanov A. I. Population model with nonlinear diffusion. // Mathematical modeling. -M., 1997, №12, p. 4356. 
